International Journal of Applied Mathematics

Volume 34 No. $6 \quad 2021,1141-1151$

ISSN: 1311-1728 (printed version); ISSN: 1314-8060 (on-line version)

doi: http://dx.doi.org/10.12732/ijam.v34i6.7

\title{
MODIFIED TWO STEPS IMPLICIT MIDPOINT ITERATION METHOD TO APPROXIMATE FIXED POINTS FOR NONEXPANSIVE MAPPINGS
}

\author{
S. Ithaya Ezhil Manna \\ Department of Mathematics \\ St. Joseph's College (Autonomous) \\ Affiliated to Bharathidasan University \\ Tiruchirappalli - 620002, Tamilnadu, INDIA
}

\begin{abstract}
In this paper, we establish the weak convergence of fixed points for nonexpansive mappings in the setting of uniformly convex Banach spaces using modified two steps implicit midpoint rule.
\end{abstract}

AMS Subject Classification: 47H09, 47H10, 47J25

Key Words: fixed point; nonexpansive mapping; uniformly convex Banach space; implicit midpoint rule

\section{Introduction}

Let $K$ be a normed linear space and $F: K \rightarrow K$ a given mapping. Let $x_{0} \in K$ be arbitrary and $\left\{\alpha_{n}\right\}$ a sequence of real numbers in $[0,1]$. The sequence $\left\{x_{n}\right\}$ is defined by

$$
x_{n+1}=\left(1-\alpha_{n}\right) x_{n}+\alpha_{n} F\left(\frac{x_{n}+x_{n+1}}{2}\right) \text {. }
$$

This implicit midpoint rule was defined by Alghamdi et al. [7] in 2014 to approximate fixed point for the nonexpansive mapping $F$ and for more results refer [6], [3], [9].

Received: May 11, 2021

(C) 2021 Academic Publications 
Here we extend equation (1) to two steps iteration scheme as

$$
\left.\begin{array}{c}
y_{n+1}=\left(1-\alpha_{n}\right) y_{n}+\alpha_{n} F\left(\frac{y_{n}+z_{n}}{2}\right) \\
z_{n}=\left(1-\beta_{n}\right) y_{n}+\beta_{n} F\left(\frac{y_{n}+y_{n+1}}{2}\right), n \geq 0
\end{array}\right\},
$$

where $\left\{\alpha_{n}\right\}$ and $\left\{\beta_{n}\right\}$ are sequences in $[0,1]$, and using this iteration scheme we shall prove the weak convergence fixed point theorem.

\section{Preliminaries}

Here we give some important results which are needed for our main results.

Lemma 1. ([8]) Let $K$ be a normed linear space and $t \in[0,1]$. Then for each $x, y \in K$,

$$
\|t x+(1-t) y\|^{2} \leq t\|x\|^{2}+(1-t)\|y\|^{2}
$$

Lemma 2. ([2]) A Banach space $K$ is uniformly convex if and only if there exists a continuous strictly increasing convex mapping $g:[0, \infty) \rightarrow[0, \infty)$ with $g(0)=0$ such that

$$
\|t x+(1-t) y\|^{2} \leq t\|x\|^{2}+(1-t)\|y\|^{2}-t(1-t) g\left(\|x-y\|^{2}\right),
$$

for all $x, y \in B_{r}[0]=\{x \in K:\|x\| \leq r\} \quad$ and $\quad 0 \leq t \leq 1$.

Lemma 3. ([4]) Let $g:[0, \infty) \rightarrow[0, \infty)$ with $g(0)=0$ be a strictly increasing mapping. If a sequence $\left\{x_{n}\right\}$ in $[0, \infty)$ satisfies $\lim _{n \rightarrow \infty} g\left(x_{n}\right)=0$, then $\lim _{n \rightarrow \infty} x_{n}=0$.

Lemma 4. ([5]) Let $\left\{x_{n}\right\}$ and $\left\{y_{n}\right\}$ are two sequences of non-negative numbers such that $x_{n+1} \leq x_{n}+y_{n}$ for all $n \geq 1$.

If $\sum_{n=1}^{\infty} y_{n}$ converges, then $\lim _{n \rightarrow \infty} x_{n}$ exists. 


\section{Main Results}

Lemma 5. Let $K$ be a nonempty bounded closed and convex subset of a uniformly convex Banach space $X$ and let $F: K \rightarrow K$ be a nonexpansive mapping such that Fix $(F):=\{x: F x=x\} \neq \emptyset$. Suppose $\left\{y_{n}\right\}$ is given by $(2)$. Then $\lim _{n \rightarrow \infty}\left\|y_{n}-p\right\|$ exists for all $p \in \operatorname{Fix}(F)$.

Proof. For any $p \in \operatorname{Fix}(F)$, we have

$$
\begin{aligned}
& \left\|y_{n+1}-p\right\|^{2}=\left\|\left(1-\alpha_{n}\right) y_{n}+\alpha_{n} F\left(\frac{y_{n}+z_{n}}{2}\right)-p\right\|^{2} \\
& \leq\left(1-\alpha_{n}\right)\left\|y_{n}-p\right\|^{2}+\alpha_{n}\left\|F\left(\frac{y_{n}+z_{n}}{2}\right)-p\right\|^{2} \\
& \leq\left(1-\alpha_{n}\right)\left\|y_{n}-p\right\|^{2}+\alpha_{n}\left\|\frac{y_{n}+z_{n}}{2}-p\right\|^{2} \\
& \leq\left(1-\alpha_{n}\right)\left\|y_{n}-p\right\|^{2}+\frac{\alpha_{n}}{2}\left\|y_{n}-p\right\|^{2}+\frac{\alpha_{n}}{2}\left\|z_{n}-p\right\|^{2} \\
& \leq\left(1-\frac{\alpha_{n}}{2}\right)\left\|y_{n}-p\right\|^{2} \\
& +\frac{\alpha_{n}}{2}\left\|\left(1-\beta_{n}\right) y_{n}+\beta_{n} F\left(\frac{y_{n}+y_{n+1}}{2}\right)-p\right\|^{2} \text {. } \\
& \left\|y_{n+1}-p\right\|^{2} \leq\left(1-\frac{\alpha_{n}}{2}\right)\left\|y_{n}-p\right\|^{2}+\frac{\alpha_{n}}{2}\left(1-\beta_{n}\right)\left\|y_{n}-p\right\|^{2} \\
& +\frac{\alpha_{n} \beta_{n}}{2}\left\|F\left(\frac{y_{n}+y_{n+1}}{2}\right)-p\right\|^{2} \text {. } \\
& \left\|y_{n+1}-p\right\|^{2} \leq\left(1-\frac{\alpha_{n} \beta_{n}}{2}\right)\left\|y_{n}-p\right\|^{2}+\frac{\alpha_{n} \beta_{n}}{2}\left\|\frac{y_{n}+y_{n+1}}{2}-p\right\|^{2} \\
& \leq\left(1-\frac{\alpha_{n} \beta_{n}}{2}\right)\left\|y_{n}-p\right\|^{2}+\frac{\alpha_{n} \beta_{n}}{4}\left\|y_{n}-p\right\|^{2} \\
& +\frac{\alpha_{n} \beta_{n}}{4}\left\|y_{n+1}-p\right\|^{2} \text {. } \\
& \left\|y_{n+1}-p\right\|^{2} \leq\left(1-\frac{\alpha_{n} \beta_{n}}{4}\right)\left\|y_{n}-p\right\|^{2}+\frac{\alpha_{n} \beta_{n}}{4}\left\|y_{n+1}-p\right\|^{2} .
\end{aligned}
$$

That is,

$$
\left(1-\frac{\alpha_{n} \beta_{n}}{4}\right)\left\|y_{n+1}-p\right\|^{2} \leq\left(1-\frac{\alpha_{n} \beta_{n}}{4}\right)\left\|y_{n}-p\right\|^{2}
$$


This implies

$$
\left\|y_{n+1}-p\right\|^{2} \leq\left\|y_{n}-p\right\|^{2}, \text { for all } n \geq 0 .
$$

Thus, the sequence $\left\{\left\|y_{n}-p\right\|\right\}$ is non-increasing and bounded.

Hence, $\lim _{n \rightarrow \infty}\left\|y_{n}-p\right\|$ exists.

Theorem 6. Let $K$ be a nonempty bounded closed and convex subset of a uniformly convex Banach space $X$ and let $F: K \rightarrow K$ be a nonexpansive mapping with Fix $(F) \neq \emptyset$. Assume $\left\{y_{n}\right\}$ is given by (2), where $\left\{\alpha_{n}\right\},\left\{\beta_{n}\right\}$ satisfy either:

(C) $0<\epsilon \leq \alpha_{n} \leq 1$ and $0<\epsilon \leq \beta_{n}\left(1-\beta_{n}\right)$ (or)

(D) $0<\epsilon \leq \alpha_{n}\left(1-\alpha_{n}\right)$.

Then $\lim _{n \rightarrow \infty}\left\|y_{n}-F y_{n}\right\|=0$.

Proof. Let $p \in F i x(T)$. Then we have

$$
\begin{aligned}
\left\|y_{n+1}-p\right\|^{2} & =\left\|\left(1-\alpha_{n}\right) y_{n}+\alpha_{n} F\left(\frac{y_{n}+z_{n}}{2}\right)-p\right\|^{2} \\
\leq & \left(1-\alpha_{n}\right)\left\|y_{n}-p\right\|^{2}+\alpha_{n}\left\|F\left(\frac{y_{n}+z_{n}}{2}\right)-p\right\|^{2} \\
& -\alpha_{n}\left(1-\alpha_{n}\right) g\left(\left\|y_{n}-F\left(\frac{y_{n}+z_{n}}{2}\right)\right\|\right) \\
\left\|y_{n+1}-p\right\|^{2} & \leq\left(1-\alpha_{n}\right)\left\|y_{n}-p\right\|^{2}+\alpha_{n}\left\|\frac{y_{n}+z_{n}}{2}-p\right\|^{2} \\
& -\alpha_{n}\left(1-\alpha_{n}\right) g\left(\left\|y_{n}-F\left(\frac{y_{n}+z_{n}}{2}\right)\right\|\right) \\
& \leq\left(1-\frac{\alpha_{n}}{2}\right)\left\|y_{n}-p\right\|^{2}+\frac{\alpha_{n}}{2}\left\|z_{n}-p\right\|^{2} \\
& -\alpha_{n}\left(1-\alpha_{n}\right) g\left(\left\|y_{n}-F\left(\frac{y_{n}+z_{n}}{2}\right)\right\|\right) \\
& \leq\left(1-\frac{\alpha_{n}}{2}\right)\left\|y_{n}-p\right\|^{2} \\
& +\frac{\alpha_{n}}{2}\left\|\left(1-\beta_{n}\right) y_{n}+\beta_{n} F\left(\frac{y_{n}+y_{n+1}}{2}\right)-p\right\|^{2} \\
& -\alpha_{n}\left(1-\alpha_{n}\right) g\left(\left\|y_{n}-F\left(\frac{y_{n}+z_{n}}{2}\right)\right\|\right)
\end{aligned}
$$




$$
\begin{aligned}
\leq & \left(1-\frac{\alpha_{n}}{2}\right)\left\|y_{n}-p\right\|^{2}+\frac{\alpha_{n}\left(1-\beta_{n}\right)}{2}\left\|y_{n}-p\right\|^{2} \\
& +\frac{\alpha_{n} \beta_{n}}{2}\left\|F\left(\frac{y_{n}+y_{n+1}}{2}\right)-p\right\|^{2} \\
& -\frac{\alpha_{n} \beta_{n}\left(1-\beta_{n}\right)}{2} g\left(\left\|y_{n}-F\left(\frac{y_{n}+y_{n+1}}{2}\right)\right\|\right) \\
& -\alpha_{n}\left(1-\alpha_{n}\right) g\left(\left\|y_{n}-F\left(\frac{y_{n}+z_{n}}{2}\right)\right\|\right) . \\
\left\|y_{n+1}-p\right\|^{2} & \leq\left(1-\frac{\alpha_{n} \beta_{n}}{4}\right)\left\|y_{n}-p\right\|^{2}+\frac{\alpha_{n} \beta_{n}}{4}\left\|y_{n+1}-p\right\|^{2} \\
& -\frac{\alpha_{n} \beta_{n}\left(1-\beta_{n}\right)}{2} g\left(\left\|y_{n}-F\left(\frac{y_{n}+y_{n+1}}{2}\right)\right\|\right) \\
& -\alpha_{n}\left(1-\alpha_{n}\right) g\left(\left\|y_{n}-F\left(\frac{y_{n}+z_{n}}{2}\right)\right\|\right) .
\end{aligned}
$$

That is,

$$
\begin{aligned}
\left(1-\frac{\alpha_{n} \beta_{n}}{4}\right) & \times\left\|y_{n+1}-p\right\|^{2} \\
& \leq\left(1-\frac{\alpha_{n} \beta_{n}}{4}\right)\left\|y_{n}-p\right\|^{2} \\
& -\frac{\alpha_{n} \beta_{n}\left(1-\beta_{n}\right)}{2} g\left(\left\|y_{n}-F\left(\frac{y_{n}+y_{n+1}}{2}\right)\right\|\right) \\
& -\alpha_{n}\left(1-\alpha_{n}\right) g\left(\left\|y_{n}-F\left(\frac{y_{n}+z_{n}}{2}\right)\right\|\right) .
\end{aligned}
$$

This implies that

$$
\begin{aligned}
\left\|y_{n+1}-p\right\|^{2} & \leq\left\|y_{n}-p\right\|^{2} \\
& -\frac{2 \alpha_{n} \beta_{n}\left(1-\beta_{n}\right)}{4-\alpha_{n} \beta_{n}} g\left(\left\|y_{n}-F\left(\frac{y_{n}+y_{n+1}}{2}\right)\right\|\right) \\
& -\frac{4 \alpha_{n}\left(1-\alpha_{n}\right)}{4-\alpha_{n} \beta_{n}} g\left(\left\|y_{n}-F\left(\frac{y_{n}+z_{n}}{2}\right)\right\|\right) .
\end{aligned}
$$

Now we rewrite the above equation into the following two forms:

$$
\begin{gathered}
\frac{2 \alpha_{n} \beta_{n}\left(1-\beta_{n}\right)}{4-\alpha_{n} \beta_{n}} g\left(\left\|y_{n}-F\left(\frac{y_{n}+y_{n+1}}{2}\right)\right\|\right) \leq\left\|y_{n}-p\right\|^{2}-\left\|y_{n+1}-p\right\|^{2}, \\
\frac{4 \alpha_{n}\left(1-\alpha_{n}\right)}{4-\alpha_{n} \beta_{n}} g\left(\left\|y_{n}-F\left(\frac{y_{n}+z_{n}}{2}\right)\right\|\right) \leq\left\|y_{n}-p\right\|^{2}-\left\|y_{n+1}-p\right\|^{2} .
\end{gathered}
$$


Case (i) Assume condition (A) holds.

From equation (3),

$$
\begin{aligned}
\frac{\alpha_{n} \beta_{n}\left(1-\beta_{n}\right)}{2} & \times g\left(\left\|y_{n}-F\left(\frac{y_{n}+y_{n+1}}{2}\right)\right\|\right) \\
& \leq \frac{2 \alpha_{n} \beta_{n}\left(1-\beta_{n}\right)}{4-\alpha_{n} \beta_{n}} g\left(\left\|y_{n}-F\left(\frac{y_{n}+y_{n+1}}{2}\right)\right\|\right) \\
& \leq\left\|y_{n}-p\right\|^{2}-\left\|y_{n+1}-p\right\|^{2} .
\end{aligned}
$$

By condition $(\mathrm{A}), \alpha_{n} \beta_{n}\left(1-\beta_{n}\right) \geq \epsilon^{2}$. Then the above equation becomes

$$
\frac{\epsilon^{2}}{2} g\left(\left\|y_{n}-F\left(\frac{y_{n}+y_{n+1}}{2}\right)\right\|\right) \leq\left\|y_{n}-p\right\|^{2}-\left\|y_{n+1}-p\right\|^{2} .
$$

Taking sum on the first $m$ terms of the above equation, we have get

$$
\frac{\epsilon^{2}}{2} \sum_{n=0}^{m} g\left(\left\|y_{n}-F\left(\frac{y_{n}+y_{n+1}}{2}\right)\right\|\right) \leq\left\|y_{0}-p\right\|^{2}-\left\|y_{m+1}-p\right\|^{2} .
$$

It is evident that, as $m \rightarrow \infty$,

$$
\sum_{n=0}^{\infty} g\left(\left\|y_{n}-F\left(\frac{y_{n}+y_{n+1}}{2}\right)\right\|\right)<\infty
$$

which gives that

$$
\lim _{n \rightarrow \infty} g\left(\left\|y_{n}-F\left(\frac{y_{n}+y_{n+1}}{2}\right)\right\|\right)=0 .
$$

Then by Lemma 3,

$$
\lim _{n \rightarrow \infty}\left\|y_{n}-F\left(\frac{y_{n}+y_{n+1}}{2}\right)\right\|=0 \text {. }
$$

Now

$$
\begin{aligned}
\left\|y_{n+1}-y_{n}\right\|^{2} & =\left\|\left(1-\alpha_{n}\right) y_{n}+\alpha_{n} F\left(\frac{y_{n}+z_{n}}{2}\right)-y_{n}\right\|^{2} \\
& \leq \alpha_{n}\left\|y_{n}-F\left(\frac{y_{n}+z_{n}}{2}\right)\right\|^{2} \cdot \\
\left\|y_{n+1}-y_{n}\right\|^{2} & \leq \alpha_{n}\left\|y_{n}-F\left(\frac{y_{n}+y_{n+1}}{2}\right)\right\|^{2}
\end{aligned}
$$




$$
\begin{aligned}
& +\alpha_{n}\left\|F\left(\frac{y_{n}+y_{n+1}}{2}\right)-F\left(\frac{y_{n}+z_{n}}{2}\right)\right\|^{2} \cdot \\
\left\|y_{n+1}-y_{n}\right\|^{2} \leq & \alpha_{n}\left\|y_{n}-F\left(\frac{y_{n}+y_{n+1}}{2}\right)\right\|^{2}+\frac{\alpha_{n}}{2}\left\|y_{n+1}-z_{n}\right\|^{2} \\
\leq & \alpha_{n}\left\|y_{n}-F\left(\frac{y_{n}+y_{n+1}}{2}\right)\right\|^{2} \\
& +\frac{\alpha_{n}}{2}\left\|y_{n+1}-\left(1-\beta_{n}\right) y_{n}-\beta_{n} F\left(\frac{y_{n}+y_{n+1}}{2}\right)\right\|^{2} . \\
\left\|y_{n+1}-y_{n}\right\|^{2} & \leq \alpha_{n}\left\|y_{n}-F\left(\frac{y_{n}+y_{n+1}}{2}\right)\right\|^{2}+\frac{\alpha_{n}}{2}\left\|y_{n+1}-y_{n}\right\|^{2} \\
& +\frac{\alpha_{n} \beta_{n}}{2}\left\|y_{n}-F\left(\frac{y_{n}+y_{n+1}}{2}\right)\right\|^{2} \cdot
\end{aligned}
$$

That is,

$$
\begin{aligned}
\left(1-\frac{\alpha_{n}}{2}\right)\left\|y_{n+1}-y_{n}\right\|^{2} & \leq \alpha_{n}\left(1+\frac{\beta_{n}}{2}\right)\left\|y_{n}-F\left(\frac{y_{n}+y_{n+1}}{2}\right)\right\|^{2} \\
\left\|y_{n+1}-y_{n}\right\|^{2} & \leq \frac{\alpha_{n}\left(2+\beta_{n}\right)}{2-\alpha_{n}}\left\|y_{n}-F\left(\frac{y_{n}+y_{n+1}}{2}\right)\right\|^{2} \\
& \leq \alpha\left\|y_{n}-F\left(\frac{y_{n}+y_{n+1}}{2}\right)\right\|^{2},
\end{aligned}
$$

where $\alpha=\min \left\{\frac{3}{2}, \frac{\alpha_{n}\left(2+\beta_{n}\right)}{2-\alpha_{n}}\right\}$.

This implies that

$$
\lim _{n \rightarrow \infty}\left\|y_{n+1}-y_{n}\right\|=0 \text {. }
$$

Case (ii) Suppose condition (B) holds.

Then from equation (4),

$$
\begin{aligned}
\alpha_{n}\left(1-\alpha_{n}\right) & \times g\left(\left\|y_{n}-F\left(\frac{y_{n}+z_{n}}{2}\right)\right\|\right) \\
& \leq \frac{4 \alpha_{n}\left(1-\alpha_{n}\right)}{4-\alpha_{n} \beta_{n}} \\
& \times g\left(\left\|y_{n}-F\left(\frac{y_{n}+z_{n}}{2}\right)\right\|\right)
\end{aligned}
$$




$$
\leq\left\|y_{n}-p\right\|^{2}-\left\|y_{n+1}-p\right\|^{2}
$$

By condition $(\mathrm{B}), \alpha_{n}\left(1-\alpha_{n}\right) \geq \epsilon$. Then the above equation becomes

$$
\epsilon g\left(\left\|y_{n}-F\left(\frac{y_{n}+z_{n}}{2}\right)\right\|\right) \leq\left\|y_{n}-p\right\|^{2}-\left\|y_{n+1}-p\right\|^{2} .
$$

Taking sum on the first $m$ terms of the above equation, we have get

$$
\epsilon \sum_{n=0}^{m} g\left(\left\|y_{n}-F\left(\frac{y_{n}+z_{n}}{2}\right)\right\|\right) \leq\left\|y_{0}-p\right\|^{2}-\left\|y_{m+1}-p\right\|^{2} .
$$

It is evident that, as $m \rightarrow \infty$,

$$
\sum_{n=0}^{\infty} g\left(\left\|y_{n}-F\left(\frac{y_{n}+z_{n}}{2}\right)\right\|\right)<\infty
$$

which gives that

$$
\lim _{n \rightarrow \infty} g\left(\left\|y_{n}-F\left(\frac{y_{n}+z_{n}}{2}\right)\right\|\right)=0 .
$$

Then by Lemma 3,

$$
\lim _{n \rightarrow \infty}\left\|y_{n}-F\left(\frac{y_{n}+z_{n}}{2}\right)\right\|=0
$$

Now,

$$
\begin{aligned}
\left\|y_{n+1}-y_{n}\right\|^{2} & =\left\|\left(1-\alpha_{n}\right) y_{n}+\alpha_{n} F\left(\frac{y_{n}+z_{n}}{2}\right)-y_{n}\right\|^{2} \\
& \leq \alpha_{n}\left\|y_{n}-F\left(\frac{y_{n}+z_{n}}{2}\right)\right\|^{2} \cdot \\
\left\|y_{n+1}-y_{n}\right\|^{2} & \leq\left\|y_{n}-F\left(\frac{y_{n}+z_{n}}{2}\right)\right\|^{2} \cdot
\end{aligned}
$$

This implies that

$$
\lim _{n \rightarrow \infty}\left\|y_{n+1}-y_{n}\right\|=0
$$

Finally,

$$
\begin{aligned}
\left\|y_{n}-F y_{n}\right\| & \leq\left\|y_{n}-F\left(\frac{y_{n}+y_{n+1}}{2}\right)\right\| \\
& +\left\|F\left(\frac{y_{n}+y_{n+1}}{2}\right)-F y_{n}\right\|
\end{aligned}
$$




$$
\begin{aligned}
& \leq\left\|y_{n}-F\left(\frac{y_{n}+y_{n+1}}{2}\right)\right\|+\frac{1}{2}\left\|y_{n+1}-y_{n}\right\| . \\
& \rightarrow \quad 0 \text { as } n \rightarrow \infty .
\end{aligned}
$$

Hence the theorem is proven.

Theorem 7. Let $K$ be a nonempty bounded closed and convex subset of a uniformly convex Banach space $X$ and let $F: K \rightarrow K$ be a nonexpansive mapping with Fix $(F) \neq \emptyset$. Assume $\left\{y_{n}\right\}$ is given by $(2)$, where $\left\{\alpha_{n}\right\},\left\{\beta_{n}\right\}$ satisfy either:

(C) $0<\epsilon \leq \alpha_{n} \leq 1$ and $0<\epsilon \leq \beta_{n}\left(1-\beta_{n}\right)$ (or)

(D) $0<\epsilon \leq \alpha_{n}\left(1-\alpha_{n}\right)$.

Then $\left\{y_{n}\right\}$ converges weakly to some fixed point of $F$.

Proof. From Lemma(5), it is clear that the sequence $\left\{y_{n}\right\}$ is bounded. Suppose there is a subsequence $\left\{y_{n_{i}}\right\}$ of $\left\{y_{n}\right\}$ such that $y_{n_{i}} \rightarrow p$ as $i \rightarrow \infty$, where $p \in F i x(F)$. Then

$$
\frac{y_{n_{i}}+y_{n_{i}+1}}{2} \rightarrow p \quad \text { as } \quad i \rightarrow \infty
$$

By the definition of continuity of $F$,

$$
F\left(\frac{y_{n_{i}}+y_{n_{i}+1}}{2}\right) \rightarrow p \text { as } i \rightarrow \infty
$$

From equation (2),

$$
z_{n_{i}}=\left(1-\beta_{n_{i}}\right) y_{n_{i}}+\beta_{n_{i}} F\left(\frac{y_{n_{i}}+y_{n_{i}+1}}{2}\right) \rightarrow p \quad \text { as } \quad i \rightarrow \infty,
$$

and

$$
y_{n_{i}}=\left(1-\alpha_{n_{i}}\right) y_{n_{i}}+\alpha_{n_{i}} F\left(\frac{z_{n_{i}}+y_{n_{i}}}{2}\right) \rightarrow p \quad \text { as } \quad i \rightarrow \infty .
$$

Continuing the above process, by induction we have

$$
y_{n_{i}+m} \rightarrow p, \text { for any } m \geq 0 .
$$

The remaining part of the proof immediately follows from Theorem 2.3 of [1].

Example 8. Let $K$ be the closed unit ball in the Hilbert space $R$ and let $F: K \rightarrow K$ defined by $F x=\frac{x}{2}$. Then clearly $F$ is nonexpansive mapping and $K$ is bounded convex subset of $R$. 
By Theorem 7, the sequence $\left\{y_{n}\right\}$ converges weakly to the fixed point of $F$. By choosing $\alpha_{n}=\frac{n}{n+1}$ and $\beta_{n}=\frac{n}{2 n+1}$, condition (B) is satisfied. Equation (2) can be equivalently written as

$$
y_{n+1}=\left(\frac{16-8 \alpha_{n}-3 \alpha_{n} \beta_{n}}{16-\alpha_{n} \beta_{n}}\right) y_{n}, \quad n \geq 0 .
$$

If we start from $y_{0}=0.5$, we obtain $y_{1}=1, y_{2}=0.363, y_{3}=0.228$, $y_{4}=0.131, y_{5}=0.071, y_{6}=0.037, y_{7}=0.019, y_{8}=0.009$, $y_{9}=0.004, y_{10}=0.002, y_{11}=0.001$ and $y_{12}=0$.

Hence the sequence $\left\{y_{n}\right\}$ converges weakly to 0 , which is clearly a fixed point of $F$ with the parameter sequences $\alpha_{n}=\frac{n}{n+1}$ and $\beta_{n}=\frac{n}{2 n+1}$.

\section{References}

[1] A.R. Khan, H. Fukhar-ud-din, Weak convergence of Ishikawa Iterates for nonexpansive maps, Proc. Wo. Co. Engg. Com. Sci., 2 (2010), 20-22.

[2] H.K. Xu, Inequalities in Banach spaces with applications, Nonlinear Anal., 16 (1991), 1127-1138.

[3] H.K. Xu, M.A. Alghamdi, N. Shahzad, The viscosity technique for the implicit midpoint rule of nonexpansive mappings in Hilbert spaces, Fixed Point Theo. Appl., 2015, No 41 (2015).

[4] H.Y. Zhou, G.T. Guo, H.J. Hwang, Y.J. Cho, On the iterative methods for nonlinear operator equations in Banach spaces, PanAmer. Math. J., 14, No 4 (2004), 61-68.

[5] K.K. Tan, H.K. Xu, Approximating fixed points of nonexpansive mappings by Ishikawa iteration process, J. Math. Anal. Appl., 178 (1993), 301-308.

[6] M.O. Aibinu, J.K. Kim, On the rate of convergence of viscosity implicit iterative algorithms, Nonlinear Funct. Anal. Appl., 25, No 1 (2020), 135152.

[7] M.A. Alghamdi, M. Ali Alghamdi, N. Shahzad, H.K. Xu, The implicit midpoint rule for nonexpansive mappings, Fixed Point Theo. Appl., 96 (2014), 1-9. 
[8] Y. Song, Q. Li, Successive approximations for quasi firmly type nonexpansive mappings, Math. Commun., 16 (2011), 251-264.

[9] Y. Yao, N. Shahzad, Y.C. Liou, Modified semi-implicit midpoint rule for nonexpansive mappings, Fixed Point Theo. Appl., 2015, No 166 (2015). 
